\title{
Das ,Schutzhaftlager' Schloss Osterstein und seine Rolle bei der Machtkonsolidierung der Nationalsozialisten im sächsischen Zwickau
}

\author{
von \\ FLORIAN HEINRICH
}

Ich erkläre hiermit ausdrücklich, dass ich im Falle meiner Entlassung aus der Verwabrungshaft mich verpflichte, mich der neuen Regierung gegenüber stets loyal zu verhalten, insbesondere mich jeder Agitation im Sinne der marxistischen Parteien zu enthalten. Ausserdem bescheinige ich, dass ich keine Klagen über Behandlung und Verpflegung während der über mich verhängten Verwabrungshaft sowie überhaupt wegen deren Verhängung und Durchfübrung zu erbeben habe und auf etwaige Ansprüche aus Anlass meiner Verwabrung verzichte. Schliesslich erkläre ich, mir bewusst zu sein, dass jeglicher Verstoss gegen diese Verpflichtungserklärung meine erneute Verwabrung zur Folge haben kann.

Zwickau, den 15. Juli $1933^{1}$

Textdokumente wie dieses beschreiben den Sinn und Zweck der durch die Nationalsozialisten tausendfach gegen Oppositionelle verhängten ,Schutzhaft' ${ }^{`}$. Das Ziel der ,Erziehung zur Volksgemeinschaft ${ }^{` 2}$ findet sich in der nationalsozialistischen Maßnahmenpolitik direkt nach der Machtübertragung 1933 wieder. Angst und Schrecken zu verbreiten, offen mit der Ausschaltung all derer zu drohen, die sich dem neuen Regime, seiner Ideologie sowie seinen menschenverachtenden Zielen zu widersetzen versuchten, war ein wesentlicher Baustein der Machtkonsolidierung der nationalsozialistischen Bewegung.

Insbesondere das Jahr 1933 war geprägt von einem gnadenlosen und umfassenden Vorgehen gegen tatsächliche und vermeintliche Gegner der Nationalsozialisten, allen voran Juden, Kommunisten und Sozialdemokraten. So waren auch Sachsen und das in dieser Arbeit im Vordergrund stehende Zwickau nur kurze Zeit nach dem 30. Januar 1933 betroffen vom Terror durch den „blutberauschte[n] Pöbel“, vom „wilde[n] unrasierte[n] Mob, nächtlich in Wohnungen einbrechend und Wehrlose in irgendwelche Folterkeller schleppend." 3 Die ersten Konzentrationslager - die in den Akten wahlweise als eben solche, als ,Schutzhaftlager" oder auch als Verwahrungslager bezeichnet werden - gaben gewissermaßen ein „Exerzierfeld für ein Unrechtsregime ab, sadistische Mißhandlungen sowie Mord und Totschlag an wehrlosen Gefangenen zu verüben."4

1 Sächsisches Staatsarchiv - Staatsarchiv Chemnitz (im Folgenden: StA Chemnitz), 30063 Geheime Staatspolizei (Staatspolizeistelle Chemnitz), Nr. 36. Anmerkung: Die in den zitierten Quellen verwendete Sprache mitsamt Abweichungen von der aktuellen Rechtschreibung wird beibehalten.

2 Titel der gleichnamigen Studie von Carina Baganz.

3 Beide Zitate: Sebastian Haffner, Geschichte eines Deutschen. Die Erinnerungen 1914-1933, München 2008, S. 123 f.

4 Klaus Drobisch/GÜNTER Wieland, System der NS-Konzentrationslager 1933-1939, Berlin 1993, S. 7. 
Von Zwickau aus, der „sächsischen Hochburg des Nationalsozialismus“, 5 begann die nationalsozialistische Bewegung, sich in Sachsen auszubreiten. Carina Baganz stellt ein frühes Bekenntnis der sächsischen Bevölkerung zur NS-Bewegung fest. ${ }^{6}$ „Gerade in Sachsen“, so Clemens Vollnhals, hatte sich „im verunsicherten Mittelstand ein massiver Antisemitismus ausgebreitet“ und damit den Boden für „populistische Parolen und antisemitische Ressentiments" " bereitet. Organisationen wie der Alldeutsche Verband entsprachen der völkischen und antisemitischen Denkschule, die seit dem Vormarsch biologistischer und rassistischer Theoriegebäude gegen Ende des 19. Jahrhunderts einen steten Zuwachs ihrer Anhänger verzeichnen konnte. Und dennoch, so stellt Andreas Wagner fest, „wurden die ersten Ortsgruppen in Sachsen im bisherigen ,Stammland der Sozialdemokratie ' gegründet ", 8 sei der Freistaat ursprünglich gar eine „Bastion der Sozialdemokratie“ gewesen, „die über eine umfangreiche Vereinskultur Milieustrukturen ausgebildet hatte. Sie konnte hier auf eine breite Arbeiterschaft zurückgreifen, die seit der in den 1850 er Jahren einsetzenden Industrialisierung ständig angewachsen war."9

Die völkische Bewegung in Sachsen erhielt nach dem Ersten Weltkrieg dennoch einigen Auftrieb. Die in der Bevölkerung unerwartete Kriegsniederlage 1918 inklusive des Zusammenbruchs von Kaiserreich und sächsischem Königreich sowie der Krisencharakter der 1920er- und frühen 1930er-Jahre waren konstituierend für die Beseitigung der Weimarer Demokratie. Infolge des chronischen Rohstoffmangels in der Mitte Europas war die sächsische Ökonomie „sowohl auf die Einfuhr von Rohstoffen als auch auf den Export weiter verarbeiteter Produkte angewiesen. Krisenhafte Schwankungen der Weltwirtschaft mußten den Freistaat daher ungleich stärker treffen als andere Regionen des Reiches."10

Die große Anzahl der Verhaftungen von NS-Gegnern in Zwickau führte Anfang März 1933 zur Einrichtung eines ,Schutzhaftlagers` im Schloss Osterstein, das die Stadtgeschichte Zwickaus bis dato über Jahrhunderte hinweg geprägt hatte. Seine Nutzung als Zuchthaus begann bereits im Jahr 1775, weshalb die Voraussetzungen für notwendigen Haftraum vorhanden waren. Karl May und August Bebel gehörten im Laufe der 187 Jahre andauernden Nutzung als Gefängnis zu den bekanntesten Insassen. Schloss Osterstein, um das Jahr 1292 einer der Siedlungsschwerpunkte Zwickaus, durchlief „im Laufe der Jahrhunderte verschiedene Phasen der Nutzung, so unter anderem als Quartier und Herberge für Truppen und Reisende [wie beispielsweise Martin Luther und August den Starken] oder als Münzstätte, stets jedoch mit einer wichtige[n] fortifikatorische[n] Funktion im ehemaligen Stadtbefestigungsring. "11

Die vorliegende Studie erfragt die Rolle, die das ,Schutzhaftlager' Schloss Osterstein bei der Machtkonsolidierung der Nationalsozialisten in Zwickau und Umgebung gespielt hat. Die Recherche zur Geschichte der (frühen) Konzentrationslager im nationalsozialistisch regierten Deutschland führt zur Aufdeckung diverser Ungleichge-

5 Ebd., S. 31.

6 Vgl. ebd., S. 11.

7 Beide: Clemens Vollnhals, Der gespaltene Freistaat: Der Aufstieg der NSDAP in Sachsen, in: Ders. (Hg.), Sachsen in der NS-Zeit, Leipzig 2002, S. 9-40, hier S. $10 \mathrm{f}$.

8 Andreas Wagner, „Machtergreifung“ in Sachsen. NSDAP und staatliche Verwaltung 1930-1935, Köln 2004, S. 31.

9 Beide: ebd., S. $31 \mathrm{f}$.

10 Ebd., S. 32.

11 Matthias Fleischhauer/Norbert Oelsner, Zum Schicksal bedeutender Baudenkmale in Zwickau. Die Priesterhäuser, Schloss Osterstein und das Kornhaus, in: Sächsische Heimatblätter 46 (2000), S. 302-318, hier S. 308. 
wichte bezüglich der Quellenlage. So verlaufen zwischen Kenntnis und Unkenntnis teils scharfe Linien, die unter anderem regional und chronologisch bedingt sind. So stellt Clemens Vollnhals „eine Fülle der regional- und lokalgeschichtlichen Studien [fest], die in der (alten) Bundesrepublik seit den Siebzigerjahren zur Erforschung der NS-Diktatur vor Ort entstanden sind“, 12 während für das Gebiet der DDR viele große Forschungslücken bestünden. In Anbetracht der Tatsache, dass eine politische Färbung der ostdeutschen Forschungslandschaft erst nach der deutschen Wiedervereinigung neutralisiert werden konnte, erscheint dieser Mangel an seriösen Studien durchaus plausibel: „Erst der Untergang der SED-Diktatur machte den Weg frei für eine differenzierte Sichtweise - fernab überkommener ideologischer Klischees und legitimatorischer Zwänge“.13 In der DDR, so der Zwickauer Historiker Norbert Peschke, habe eine staatlich verordnete Fokussierung auf einige wenige Opfergruppen, das beinhaltet vor allem Kommunisten und wenige Sozialdemokraten, eine umfassende Auseinandersetzung mit der Situation des Jahres 1933 erheblich erschwert. Zudem wurden viele Dokumente erst nach der Wiedervereinigung zur Einsicht freigegeben. Auch Baganz verweist auf diese besondere Forschungssituation, wenn sie „die Geschichte der kommunistischen Häftlinge im Zentrum der [ostdeutschen] Untersuchungen“ 14 verortet. Kurzum: „Die Geschichte der Konzentrationslager wurde in der DDR überwiegend als Teil der Geschichte der KPD und des kommunistischen Widerstandes geschrieben." 15 Umso mehr sei darauf hingewiesen, dass zehn von zwölf zum Zwecke dieser Arbeit ausgewerteten Akten aus dem Staatsarchiv Chemnitz Aufschluss über das Schicksal von Mitgliedern der Sozialdemokratischen Partei geben.

Das Aufholen nach der Wiedervereinigung finde sichtbar statt, habe jedoch mit einem zentralen Problem zu kämpfen, namentlich der „sehr schlechten Quellenlage in den Archiven“, 16 die auch bei der Erarbeitung dieser Studie Einschränkungen mit sich

gebracht hat. Ferner diagnostiziert Carina Baganz Forschungslücken vor allem im Bereich der frühen Konzentrationslager, „die unmittelbar nach der Machtübernahme der Nationalsozialisten im Frühjahr 1933 wie Pilze aus dem Boden schossen. “17 Zu dieser frühen Kategorie der nationalsozialistischen Lager gehört auch das im Mittelpunkt stehende ,Schutzhaftlager' Schloss Osterstein, das vermutlich vom März 1933 bis Ende Januar 1934 als solches bestand. Ab Februar 1934 erfolgte die Ausgliederung der Schutzhäftlinge auf die Sachsenburg und das Schloss wurde wieder als Zuchthaus betrieben. Beispielsweise verglichen mit der Sachsenburg sind die Erkenntnisse über das KZ Schloss Osterstein eher gering und schon gar nicht in gebündelter Form einsehbar. Generell gebe es ,aus neuerer Zeit kaum eine zusammenfassende Abhandlung "18 die regionalspezifischen Umstände der nationalsozialistischen Machterringung und Konsolidierung in Zwickau und Umgebung betreffend.

12 Clemens Vollnhals, Vorbemerkung, in: Ders., Sachsen in der NS-Zeit (wie Anm. 7), S. 7.

13 Ebd.

14 Carina Baganz, Erziehung zur „Volksgemeinschaft“? Die frühen Konzentrationslager in Sachsen 1933-34/37 (Geschichte der Konzentrationslager 1933-1945 6), Berlin 2005, S. 14.

15 Ebd.

16 Ebd.

17 Ebd., S. 12.

18 Norbert Peschke (Hg.), Naziterror in Zwickau. Über Widerstand und Verfolgung politischer Gegner während des NS-Regimes in der Zwickauer Region (1933-1939), Zwickau 2005, S. 7. 
Ein weiterer Erklärungsansatz für die rare Zahl der Quellen liegt in der Frühzeitigkeit der im Vordergrund stehenden Lager im Gesamtbild der zwölf Jahre Nationalsozialismus. In diesem zeitlichen Rahmen machen sie einen geringeren Anteil aus und sind die Ausmaße der Inhaftierungen - ohne jede Absicht der Verharmlosung - mit denen in den späteren Terror- und Vernichtungslagern nicht gleichzusetzen. Auch deshalb redet die Öffentlichkeit und Forschungslandschaft heute eher von Buchenwald und Auschwitz statt von Zwickau oder Hohnstein. ${ }^{19}$

Darüber hinaus wurde in Lagern, deren Bestandsdauer von vornherein nur auf wenige Wochen oder Monate ausgerichtet war, nicht unbedingt eine eingehende Dokumentation der dortigen Verhältnisse unternommen. ${ }^{20}$ Bestätigung erhält diese Vermutung durch Feststellungen bei der Einsicht in Akten des Staatsarchivs Chemnitz. So sind die verwendeten Akten nicht etwa im Bestand des Zuchthauses Zwickau zu finden, sondern in dem der Gestapo-Stelle Chemnitz. In deren Zuständigkeit fielen die betroffenen Personen vermutlich aufgrund der noch jahrelang betriebenen offenen wie auch geheimen Nachüberwachung. Gewiss erfolgte aus Kapazitätsgründen eine Beschränkung auf zwölf Akten über inhaftierte Personen im Schloss Osterstein. Doch darüber hinaus wären die zusätzlich einsehbaren Akten für die hier aufgeworfene Fragestellung kaum oder gar nicht von Bedeutung gewesen. Das liegt unter anderem daran, dass der betreffende Zeitraum 1933/1934 im Vergleich zu späteren Jahren sehr mäßig und im Bestand von Schloss Osterstein überhaupt nicht vertreten ist. Erwähnt werden müssen auch die bestehenden Schutzfristen für Personenakten, die eine Nennung von Klarnamen nicht zulassen.

Letztlich sind viele Dokumente „mit an Sicherheit grenzender Wahrscheinlichkeit bei den Bombenangriffen auf Dresden in den letzten Kriegswochen vernichtet wurden. Dies ist zum Beispiel für die Akten der Gauleitung Sachsen der NSDAP der Fall." 21 Diese Umstände müssen durch die historische Forschung hingenommen und durch gründliche Arbeit mit dem vorhandenen Material kompensiert werden. Doch auch die exemplarische Auswahl der zwölf erwähnten Akten mit dem Inhaftierungsdatum 25. Juni 1933 und dem Entlassungsdatum 15. Juli 1933 und angeordneter Nachüberwachungen ermöglicht zentrale Erkenntnisse über die Motive für die Verhängung sogenannter Schutzhaft auf Schloss Osterstein sowie deren Konsequenzen.

Die Haftumstände betreffend muss ein Rückgriff auf einen anderen Quellentyp erfolgen. Hier sollen vor allem drei Schriften im Vordergrund stehen. Zuallererst sei Franz Thies' Bericht über den Osterstein-Prozess des Jahres 1948 erwähnt, der unter anderem die Anklageschrift gegen einige verantwortliche Aufseher des Lagers beinhaltet. Zudem wird die Studie zum Wirken des KPD-Funktionärs Martin Hoop in Zwickau sowie Helmut Rothes Schrift zum Antifaschistischen Widerstandskampf in Zwickau herangezogen. Eine gewisse Vorsicht im Umgang mit diesen Texten gebietet sich aufgrund ihrer Einbettung in die Geschichtsschreibung in der DDR und der daraus resultierenden Interpretationen des Geschehenen.

\section{Die ,Schutzhaft' als Instrument der nationalsozialistischen Maßnabmenpolitik}

Der Reichstagsbrand in der Nacht vom 27. auf den 28. Februar 1933, für den der niederländische Kommunist Marinus van der Lubbe verantwortlich gemacht und hinge-

19 Vgl. ebd., S. 12.

20 Vgl. ebd., S. 19 f.

21 Ebd., S. 19. 
richtet wurde, gilt als „eine entscheidende Zäsur“22 auf dem Weg hin zum nationalsozialistischen Terrorstaat. „Indem die Nationalsozialisten den Rauch über dem Wallot-Bau als kommunistisches Aufstandssignal interpretierten, konnten sie lange bereitliegende Pläne umsetzen."23

Die noch am 28. Februar 1933 erlassene Reichstagsbrandverordnung beziehungsweise ,Verordnung zum Schutz von Volk und Staat' wurde alsbald zu einem „Inbegriff der politischen Gegnerbekämpfung “24 durch die Nationalsozialisten. Zwar hatte das radikale Vorgehen gegen politische Opponenten bereits kurz vor dem 30. Januar 1933 begonnen und weitete sich „unmittelbar nach der Ernennung Adolf Hitlers zum Reichskanzler“ aus, diente „zu dieser Zeit jedoch noch nicht ausschließlich der Festigung des nationalsozialistischen Systems, sondern vor allen Dingen der Rache für [vor dem 30. Januar 1933] zugefügte Schäden." ${ }^{25}$ Das gehe Adolf Diamant zufolge aus „unzähligen Dokumenten“ 26 hervor. Diese Motivlage rückte ab Ende Februar zunehmend in den Hintergrund. Von nun an lag das Hauptaugenmerk der NS-Maßnahmen auf der Sicherung und dem Ausbau der eigenen Machtposition, auch in Anbetracht der bevorstehenden Reichstagswahl am 5. März. Seit der Reichstagsbrandverordnung entstanden daher „überall in Deutschland kleinere und größere Lager, die frühen Konzentrationslager.“27 Die Verfolgung von Menschen jüdischen Glaubens, Sozialdemokraten, Kommunisten und Gewerkschaftern erreichte eine neue Qualität.

Hatte im Februar unter anderem noch die Rücksichtnahme auf den Koalitionspartner eine Rolle gespielt, kam es ab „den Märzwahlen 1933 zu großen Verhaftungsaktionen und Straßenrazzien. "28 Basierend auf der Verordnung vom 28. Februar 1933, die den Terminus der ,Schutzhaft" nicht einmal beinhaltete, wurde diese von den Nationalsozialisten zynisch so genannte Maßnahme ab dato „zum Inbegriff der politischen Gegnerbekämpfung. “29 Durch das nicht nur billigende, sondern sogar fördernde Verhalten des Reichspräsidenten Paul von Hindenburg erhielten Vorstöße verschiedenster Art gegen oppositionelle Kräfte einen pseudolegalen Anstrich.

„Die Zerschlagung der Arbeiterbewegung und ihrer Parteien“, so Wolfgang Benz und Barbara Distel, sei „das erste umfassende Ziel“30 der innenpolitischen Maßnahmen gewesen. Listen mit potenziellen Häftlingen seien Franz Thies zufolge schon im Vorfeld erstellt worden. ${ }^{31}$ Der wesentliche Unterschied zwischen Februar und März 1933: Bereits mit der am 4. Februar 1933 erlassenen „Notverordnung zum Schutz des deutschen Volkes“ wurde eine „Möglichkeit stark erweiterter Dauer polizeilicher Haft (bis zu drei Monaten)“ geschaffen, die „jedoch ausdrücklich nur bei Verdacht strafbarer Handlungen“ Anwendung fand und „dem Verhafteten außerdem das Recht

22 Magnus Brechtken (Hg.), Die nationalsozialistische Herrschaft 1933-1939, Darmstadt 2004, S. 23.

23 Ebd.

24 Baganz, Volksgemeinschaft (wie Anm. 14), S. 52.

25 Beide ebd., S. 50.

26 Adolf Diamant, Gestapo Chemnitz und die Gestapoaußenstellen Plauen i. V. und Zwickau. Zur Geschichte einer verbrecherischen Organisation in den Jahren 19331945, Chemnitz 1999, S. 28.

27 Baganz, Volksgemeinschaft (wie Anm. 14), S. 53.

28 Wolfgang Benz/Barbara Distel (Hg.), Instrumentarium der Macht. Frühe Konzentrationslager 1933-1937 (Geschichte der Konzentrationslager 1933-1945 3), Berlin 2003, S. 10.

29 Baganz, Volksgemeinschaft (wie Anm. 14), S. 52.

30 Beide Zitate: Benz/Distel, Instrumentarium der Macht (wie Anm. 28), S. 10.

31 Vgl. Franz Thies, Der Prozeß Schloss Osterstein, Zwickau 1948, S. 3. 
[zugestand], den Richter anzurufen, der im Zweifelsfall über die Fortdauer der Haft zu entscheiden hatte." ${ }^{32}$ Demnach lag eine juristische Grundlage für Inhaftierungen nur bei Straftaten wie Verrat beziehungsweise dem Verdacht dazu vor. Mit dem 28. Februar 1933 und der Reichstagsbrandverordnung wurde die ,Schutzhaft' ${ }^{\prime} \mathrm{zu}$ einer vorbeugenden Maßnahme. ${ }^{33}$ Die Aufhebung der persönlichen Freiheit schuf „die Grundlage für die polizeiliche Verhaftung von politischen Gegnern [...], die sich grundsätzlich von der auf dem Wege eines Rechtsverfahrens durch ein Gerichtsurteil begründeten Strafhaft und der nur kurzfristig erlassenen Polizeihaft unterschied."34 Weitere Merkmale dieser waren das Fehlen einer judikativen Legitimierung und von Rechtsmitteln sowie ihr „nahezu durchweg völlig unbefristet[er]“35 Charakter.

Die ungezügelte Verfolgung vermeintlicher innenpolitischer Feinde konnte von nun an endgültig seinen freien Lauf nehmen. Bis zum Ende des Februars 1933 hatte sie ihr Hauptbetätigungsfeld trotz vorheriger Übernahme der Reichsregierung noch auf der Straße gefunden. Jetzt wurde sie zum „staatlich sanktionierten Terror.“ 36 Der nun herrschende „permanente Ausnahmezustand“ 37 wurde bis zum Kriegsende zur Regel. Carsten Schreiber schildert die Verhältnisse der ersten Märztage des Jahres 1933 wie folgt: „Für kurze Zeit verlor die Polizei die Kontrolle über die Straße, und die Nationalsozialisten ergriffen die Gunst der Stunde. [...] SA-Trupps brachen Haustüren auf und verschleppten ihre Gegner. Die Polizei hielt sich angesichts der unklaren Machtverhältnisse abseits, während die Nationalsozialisten ihre Gegner prügelten, töteten und ihre Parteilokale in improvisierte Haft- und Folterstätten umwandelten. Neben dem ungezügelten Straßenterror der SA ging ab dem 1. März auch die Politische Polizei, die als eine besondere Abteilung den Polizeipräsidien zugeordnet war, gegen die KPD vor und verhaftete deren Funktionäre."38

Ergänzt wurde die Arbeit der Politischen Polizei am 3. März durch die Bildung einer 1500 Mann starken Hilfspolizei, die ihr Personal aus verlässlichen und gewaltbereiten Nationalsozialisten rekrutierte. ${ }^{39}$ Diese Maßnahmen ermöglichten eine Zahl von 8976 sächsischen Häftlingen allein bis zum 13. April 1933.40

Die infolge dieser Entwicklung entstehende und zahlenmäßig große Häftlingsgruppe der Oppositionellen stellte die für die nationalsozialistische Machtsicherung Verantwortlichen vor Herausforderungen. Zum einen musste - zusätzlich zu den ohnehin bestehenden Haftanstalten - Platz geschaffen werden, um die neuen Häftlinge unterzubringen. Allein im Jahr 1933 betraf das ungefähr 80000 Personen. ${ }^{41}$ Folglich entstanden nach einer provisorischen Phase, in der vorwiegend Parteilokale und Kellerräume zu Haft- und Folterstätten umfunktioniert wurden, fast 70 Konzentrationslager im gesamten Reichsgebiet. ${ }^{42}$ Für das ,Schutzhaftlager' Schloss Osterstein wird

32 Alle Zitate: Martin Broszat, Nationalsozialistische Konzentrationslager 1933-1945, in: Hans Buchheim u. a. (Hg.), Anatomie des SS-Staates, Bd. 2, München 1979, S. 14.

33 Vgl. ebd.

34 Ebd.

35 Drobisch/Wieland, System (wie Anm. 4), S. 29.

36 Mike Schmeitzner, Ausschaltung - Verfolgung - Widerstand. Die politischen Gegner des NS-Systems in Sachsen 1933-1945, in: Vollnhals, Sachsen in der NS-Zeit (wie Anm. 7), S. 183-199, hier S. 187.

37 BRechtKen, NS-Herrschaft (wie Anm. 22), S. 23.

38 Carsten Schreiber, Täter und Opfer: Der Verfolgungsapparat im NS-Staat, in: Vollnhals, Sachsen in der NS-Zeit (wie Anm. 7), S. 170-182, hier S. 172.

39 Vgl. ebd.

40 Vgl. Drobisch/Wieland, System (wie Anm. 4), S. 37.

41 Vgl. Benz/Distel, Instrumentarium der Macht (wie Anm. 28), S. 11.

42 Vgl. ebd. 
beispielsweise ein Zeitraum zwischen dem 1. und dem 10. März angenommen, in dem erste politische Häftlinge eingeliefert worden sind. Zum anderen brachte die schiere Anzahl der Lager und die damit einhergehende Tendenz zur mangelnden Übersicht über sie die Notwendigkeit zentraler Regelungen mit sich. So verfügte vor allem Sachsen „über eine große Zahl von Schutzhaftbestimmungen, die besonders detailliert ausformuliert waren“; 43 der Grund dafür sei Carina Baganz zufolge „in der großen Zahl der in Sachsen bestehenden frühen Konzentrationslager und in der Brutalität derselben "44 $\mathrm{zu}$ finden. Folglich informierte das Landeskriminalamt Dresden das Innenministerium am 28. März 1933 über die Einrichtung einer eigens für die Verwaltung der ,Schutzhaft' zuständigen Zentrale. Erreicht werden sollte mit dieser Maßnahme „eine gleichmäßige Behandlung aller in Sachsen in Schutzhaft genommenen Personen. " 45 Erste Amtshandlung dieser Zentrale war die Formulierung enger Richt-

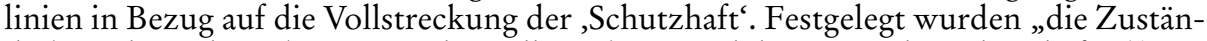
digkeit, die Zulässigkeit sowie die Vollstreckung und die Dauer der Schutzhaft. "46 Die Gefährdung nationaler Interessen durch marxistische Parteien und eine hohe Funktion in marxistischen Parteien waren hauptausschlaggebende Motive für Inhaftierungen. ${ }^{47}$ Sowohl die Mitglieder der sogenannten marxistischen Parteien und deren Organisationen als auch deren Führungskader konnten Opfer der ,Schutzhaft' werden; dass im selben Kontext auch Schwerkriminelle Erwähnung finden, zeigt den Versuch der Kriminalisierung und Stigmatisierung politischer Gegner. ${ }^{48}$

Einen weiteren Regulierungsversuch von staatlicher Stelle markierten Bestimmungen vom 19. April 1933. Ihr Inhalt waren „umfangreiche und detaillierte Instruktionen " 49 zur Behandlung der Häftlinge, was unter anderem ein Verbot der körperlichen Bestrafung sowie die Vorgabe eines strengen und nur vermeintlich gerechten Umgange mit den Insassen der jeweiligen Lager bedeutete. Am 5. August erging zu diesen Bestimmungen ein offizieller Nachtrag. Neben der Frage, welche Lager den Status eines Konzentrationslagers beziehungsweise eines Arbeitsdienstlagers erhalten sollten, wurden weitere Richtlinien die Durchführung der ,Schutzhaft' betreffend formuliert. Unter anderem sollten von nun an Fragebögen über die Zustände in den Lagern zu deren Erfassung beitragen. ${ }^{50}$ Letztlich scheiterten diese Vereinheitlichungsversuche seitens der NS-Verwaltung. Reglements zu Befugnissen, Zuständigkeiten und Durchführung der Haft „wurden in den seltensten Fällen beachtet, denn wie vielen Dokumenten und Berichten $\mathrm{zu}$ entnehmen ist, nahmen auch SA- und SS-Angehörige willkürlich Festnahmen vor, schleppten die Gefangenen in die Konzentrationslager oder Folterstätten. Taten sie es nicht selbst, verlangten sie dies von der Polizei."51 berg, Nr. 2193, unpag., abgedruckt in: BAgANZ, Volksgemeinschaft (wie Anm. 14), S. 371 .

46 Baganz, Volksgemeinschaft (wie Anm. 14), S. 72.

47 Vgl. ebd., S. 69.

48 Vgl. ebd., S. 70.

49 Die Errichtung der „frühen“ Konzentrationslager im Frühjahr/Sommer 1933 in Sachsen, hrsg. von der Stiftung Sächsische Gedenkstätten, https://www.stsg.de/cms/stsg/ ausstellungen/fruehe_kz_in_sachsen/errichtung-der-fruehen-konzentrationslager-imfruehjahr-sommer-1933-in-sachsen [Zugriff: 28. März 2016].

50 Vgl. Baganz, Volksgemeinschaft (wie Anm. 14), S. 71 f.

51 Ebd., S. 69. 
Viele ,Schutzhäftlinge‘ wurden laut Benz und Distel „im Zuge der politischen Stabilisierung des Nationalsozialismus wieder entlassen. "52 So verweisen unter anderem die eingangs erwähnten und für diese Arbeit wesentlichen Akten auf eine Entlassung mehrerer Personen aus Schloss Osterstein am 15. Juli 1933. Die ,Schutzhaft' als „eines der wichtigsten Instrumente zur Festigung der nationalsozialistischen Diktatur“ 53 hatte in diesem wie auch in vielen anderen Fällen - etwa vor der Reichstagswahl vom 5. März 1933 - ihre zentrale Aufgabe erfüllt und politische Gegner aus dem Verkehr gezogen. Im Anschluss daran wurden diese häufig durch den Verlust des Berufes, strenge Meldeauflagen sowie die Begleichung der Haftkosten zermürbt. Für den Fall Osterstein sprechen wir von 40 Reichsmark, die nur bei ausführlicher Begründung in Raten gezahlt werden konnten. Hinzu kam häufig sowohl eine offene als auch geheime Nachüberwachung der Entlassenen, welche erst durch die offizielle Feststellung beendet werden konnte, die betroffene Person füge sich in die ,Volksgemeinschaft ${ }^{`}$ ein und sei keine Gefahr für den Staat.

\section{Der Mythos von den, wilden'Lagern}

Der Improvisationscharakter vieler früher Konzentrationslager und die Kurzfristigkeit ihrer Einrichtung verleiten hin und wieder zu der Bezeichnung, wilde Lager'. Diesen Begriff charakterisiert wesentlich der mangelnde staatliche Zugriff auf die Zustände in den ersten Lagern des März 1933. Er suggeriere, so Carina Baganz, „eine durch die SA oder SS vorgenommene exzessartige Inhaftierung, ohne Kenntnis oder Unterstützung von Polizei und Verwaltung. " 54 Derartige Umstände sind jedoch eher für den Februar 1933 zu diagnostizieren, also vor der Einrichtung von Lagern, als denn einen Monat später.

Die Angaben über die Zahl der 1933 und 1934 existierenden Konzentrationslager in Deutschland schwanken je nach konsultierter Literatur zwischen knapp 60 und circa 100. Konkrete Zahlen zu den Lagern oder Haftstätten seien nicht genau zu belegen, vorwiegend aus folgendem Grund: Eine Typisierung der erfassten Haftstätten durch die NS-Verwaltung erfolgte erst im Laufe des Jahres 1933.55 So entschieden die ,Vorläufigen Bestimmungen über die Errichtung und Verwaltung von Konzentrationslagern und Arbeitsdienstlagern' (April/August 1933) darüber, welches sächsische Lager welche Bezeichnung erhielt. 56 Das ,Schutzhaftlager' Schloss Osterstein wird in diesem Zusammenhang am 5. August als Konzentrationslager, von Mitte August 1933 an gar als ,Verwahrungsanstalt' benannt, was einer begrifflichen Verharmlosung gleich kommt. ${ }^{57}$ Letzten Endes seien derartige Differenzierungen aber „nur eine Farce“ gewesen und wurden die Inhaftierten „den Lagern beliebig zugeteilt.“58

Das Zwickauer Lager war aufgrund der Bündelung politischer Gegner auf engem Raum faktisch ein Konzentrationslager. Carina Baganz rechtfertigt die Verwendung des KZ-Begriffes: „Auch wenn in den frühen Konzentrationslagern weniger Tote zu verzeichnen waren, die Inhaftierten wurden misshandelt, gepeinigt, gequält und erniedrigt, sie empfanden die Lager, die Grausamkeit und Brutalität als das Schlimmste,

52 Benz/Distel, Instrumentarium der Macht (wie Anm. 28), S. 11.

53 Baganz, Volksgemeinschaft (wie Anm. 14), S. 68.

54 Ebd., S. 54.

55 Vgl. ebd., S. 55.

56 Vgl. ebd., S. 58.

57 Vgl. ebd., S. 59 f., 71.

58 Ebd., S. 58. 
das Mörderischste, was ihnen bisher geschehen war."59 Damit wurde Schloss Osterstein zur Haftstätte für alle, „die sich als Schädlinge am deutschen Volkskörper erwiesen haben und deren Sinnesänderung insoweit aussichtslos erscheint, das sind insbesondere die Funktionäre und sonstigen Führer der marxistischen Verbände und kriminell schwer vorbestrafte Personen."60 An anderer Stelle wird gezeigt, dass eine Beeinflussung der Inhaftierten zugunsten der NS-Ideologie keinesfalls derart aussichtslos war. In verschiedenen Fällen trat bei ehemaligen ,Schutzhäftlingen` durchaus ein Sinneswandel auf, zu dessen Ursachen und Tatsächlichkeit an geeigneter Stelle die nötigen Fragen gestellt werden müssen.

Ein weiteres Argument gegen die Bezeichnung, wilde Lager' ist die klare Zielgerichtetheit und langfristige Planung der nationalsozialistischen Haftmaßnahmen gegen politische Gegner. Hitler formulierte bereits 1924 die nationalsozialistische Pflicht, die Verteidigung [des deutschen Staates] gegen den Terror der siegestrunkenen Internationale selbst zu übernehmen. ${ }^{61}$ In Kontinuität zu dieser Forderung aus „Mein Kampf“" steht eine Äußerung Wilhelm Fricks gegenüber dem SPD-Reichstagsabgeordneten Gerhart Seger vom Dezember 1932: Wenn wir zur Macht kommen, werden wir euch Kerle alle ins Konzentrationslager stecken. ${ }^{62}$ Feststellen lässt sich unter anderem durch das Heranziehen derartiger Aussagen, dass die ersten Konzentrationslager des Frühjahrs 1933 zwar durchaus das Ergebnis der Überfüllung anderer Haftstätten gewesen sein können, so auch in Zwickau, dass eine langfristige Planung der frühen Lager allerdings ebenso wenig geleugnet werden kann wie eine frühzeitig einsetzende Kooperation zwischen SA, SS auf der einen, Polizei und Verwaltung auf der anderen Seite. ${ }^{63}$

Dem Ausmaß von „Terror, Misshandlungen, Arbeit, Arrest, Folter und Mord“64 in den frühen Konzentrationslagern habe auch die langfristige Planung keinen Abbruch getan. Im Gegenteil, selbst in den strukturiertesten Lagern stoßen wir auf durchweg unmenschliche Verhältnisse.

\section{III. „Euch müßte man doch alle erschießen“ - Foltern für ein , besseres Deutschland“}

„Folter und Tortur“65 waren die wesentlichen Bestandteile des Häftlingsalltags im „Schutzhaftlager“ Schloss Osterstein gewesen. Wie eingangs erwähnt, macht der Versuch einer Schilderung der Verhältnisse im Zwickauer KZ unter anderem den Rückgriff auf in der DDR verfasste Literatur notwendig. Zwar geben die gesichteten Akten beispielsweise Einblick in Haftdauer und Nachüberwachung, jedoch nicht in Haftbedingungen im Lager selbst. Hierfür werden die entsprechenden Quellen sowie Sekun-

59 Ebd., S. 62.

60 Ebd., S. 58.

61 Adolf Hitler, Mein Kampf. Zwei Bände in einem Band, URL: http://www.harrold. org/rfhextra/download/adolf\%20hitler\% 20-\% 20mein \% $20 \mathrm{kampf} \% 20$-\%20german. pdf [Zugriff: 19. Oktober 2013].

62 Drobisch/Wieland, System (wie Anm. 4), S. 14.

63 Vgl. Baganz, Volksgemeinschaft (wie Anm. 14), S. 53 f.; und Benz/Distel, Instrumentarium der Macht (wie Anm. 28), S. 13.

64 Baganz, Volksgemeinschaft (wie Anm. 14), S. 61.

65 Helmut Rothe (Hg.), Antifaschistischer Widerstandskampf in Zwickau. Schloß Osterstein 1933 bis 1945. Außenlager des KZ Flossenbürg in Zwickau 1942 bis 1945 (Beiträge zur Geschichte der Zwickauer Arbeiterbewegung 15), Zwickau 1985, S. 21. 
därliteratur ebenso herangezogen wie eine knappe Vorstellung der baulichen Substanz von Schloss Osterstein, die für die Konstituierung des Haftraumes wesentlich ist.

Der im Frühjahr 1933 zur Verfügung stehende Raum im Schloss Osterstein gliederte sich in drei Gebäudeteile: den Schlosskomplex mit verteilten Flügeln in alle vier Himmelsrichtungen, das sogenannte Kornhaus beziehungsweise Nordgebäude und das inzwischen abgerissene Zellenhaus. ${ }^{66}$ Über die Beschaffenheit des letzteren Gebäudes informiert Gotthold Leistner. Einschränkend hierbei: Leistner bezieht sich in seiner Beschreibung auf den Zeitraum um 1870/1880. Da jedoch keine größeren Baumaßnahmen zwischen 1880 und 1933 bekannt sind und angesichts der durchgehenden Nutzung als Haftstätte auch nicht plausibel erscheinen, soll Leistners Beschreibung hier als Annäherung für die infrastrukturellen Verhältnisse des Jahres 1933/1934 gelten. Das Zellenhaus beinhaltete rund 140 Gefängniszellen in einer Größe zwischen circa 8 und 10 Quadratmetern. ${ }^{67}$ Aus Franz Thies' Bericht über den Osterstein-Prozess kann entnommen werden, dass für jede Zelle ein Häftling vorgesehen war. ${ }^{68} \mathrm{Die}$ durch die NS-Wachmannschaften forcierte Trennung politischer Gefangener voneinander legt nahe, dass dieses Vorgehen auch 1933/1934 beibehalten wurde. Bade- und Lagerräume sollen ebenso vorhanden gewesen sein wie gasbeleuchtete Korridore; die Zellen hingegen seien, wenn überhaupt, mit Öllampen erhellt worden. ${ }^{69}$ Darüber hinaus habe eine im Souterrain installierte Warmwasserbeizung 70 für Temperaturen zwischen 18 und 20 Grad Celsius gesorgt, im Winter deutlich kälter (circa 8 bis $9 \mathrm{Grad}$ Celsius).

Die Zellentüren seien nach Leistners Beschreibung mit Eisenblech beschlagen und mit Spion und einer Durchreiche [für Mablzeiten] verseben ${ }^{71}$ gewesen. Generell scheinen sich die Zellen des Zellenhauses von denen anderer Gebäudeteile unterschieden zu haben. So spricht Leistner von einer Ausstattung der Zellen mit einem Klapptisch, einem Schemel beziehungsweise Stuhl, einer Toilette sowie einem Spucknapf und einer Strohmatratze auf einem Klappbettgestell. ${ }^{72}$ Hierin scheint doch eine wesentliche Differenz zwischen den Jahren 1880 und 1933 zu liegen. Die nationalsozialistischen Verantwortlichen des Lagers scheinen das Zellenhaus umfunktioniert zu haben zu einer Unterbringung für diejenigen Häftlinge, denen eine besonders schlechte Behandlung zukommen sollte. Vor allem die sogenannten 0 - und $00-Z$ ellen müssen ein Hort der Unmenschlichkeit gewesen sein. Der Bericht eines Zeugen aus dem Prozess gegen ehemalige Aufseher des Zwickauer KZ soll hierüber Aufschluss geben. Waldemar Trommer aus Falkenstein beschreibt seine Inhaftierung im Zellenhaus wie folgt: Ich wurde von den SS-Schergen Beetz und Biedermann in die sogenannte 00-Zelle geschleppt. Diese 00-Zelle befand sich im Keller. Es war eine fensterlose Zelle und erinnerte mich an die unterirdischen Verliese der Ritterburgen, wie man sie uns in der

66 Vgl. Peschke, Naziterror (wie Anm. 18), S. 38.

67 Vgl. GotThold Leistner, Das Zellenhaus im „Schloß Osterstein“, in: Zwickauer Heimatjournal 3 (1994), S. 16-22, hier S. 19. Anmerkung: Die Zellengröße wurde berechnet auf Basis einer Umrechnung von Fuß in Meter.

68 Vgl. Thies, Prozess (wie Anm. 31), S. 7.

69 Vgl. Leistner, Zellenhaus (wie Anm. 67), S. 19.

70 Ebd.; Anmerkung: Inwiefern die Insassen der Zellen von einer Beheizung des Gebäudes und auch der Korridore profitierten, kann nicht genau festgestellt werden. An anderer Stelle berichtet ein ehemaliger Gefangener von seiner Unterbringung in einer selbst in den Sommermonaten [...] feucht[en] und kalt[en] 00-Zelle im selben Gebäude; siehe Thies, Prozess (wie Anm. 31), S. 9.

71 Ebd.

72 Vgl. Leistner, Zellenhaus (wie Anm. 67), S. 20. 
Schulzeit beschrieben hatte. Meterdicke Steinwände, aus denen Salpeter quillt. Selbst in den Sommermonaten sind die Zellen feucht und kalt. Eine ständige Dunkelheit berrscht, und die Häftlinge sind gezwungen, auf dem nackten Steinfußboden obne Strobsack, obne Matratze zu schlafen. Kein Tisch, kein Stubl, befindet sich in der Zelle, nur in einer Ecke ein Gefäß, um die Notdurft zu verrichten. Selbst am Tage sitzt der Gefangene auf dem kalten, feuchten Fußboden. ${ }^{73}$

Diese Schilderung der Isolationshaft bringt nicht nur Erkenntnisse zu den Verhältnissen im Zellenhaus, sondern zeigt indirekt, dass Zellen in anderen Trakten des Lagers durchaus mit einigem wenigen Mobiliar ausgestattet gewesen sein müssen. Andernfalls hätte der Zeuge dessen Fehlen in der erwähnten 00-Zelle wohl nicht angemerkt. Carina Baganz ergänzt in einem Aufsatz zu Schloss Osterstein, dass andere Zellen, vermutlich auch im 0- und 00-Bereich, einen ,auf dem Fußboden einbetonierte[n] Ring mit einer Kette [hatten], an die der Häftling gefesselt werden konnte. "74 Des Weiteren beschreibt sie die sanitären Zustände als „ähnlich schlimm wie die Unterbringung der Häftlinge" 75 und bezieht sich damit auf Kübeltoiletten im Zellenhaus beziehungsweise Aborte mit Fallrohren und Kübel, beispielsweise im Nordgebäude. ${ }^{76}$ Überhaupt scheint das Nordgebäude erst ab dem 14. Juli 1933 für das Gewahrsam der ,Schutzhäftlinge' genutzt worden zu sein. ${ }^{77}$

Eine weitere bedeutsame Räumlichkeit im Schloss Osterstein war der sogenannte Zandersaal. Eigens für die Folter politischer Gegner unter dem Dachstuhl des Nordgebäudes neu eingerichtet, wurde der mit lediglich einem Tisch, zwei Stühlen und einigen Matratzen ausgestattete Raum zur „Hauptfolterstätte“78 im Lager. Franz Thies berichtet von der zynischen Bezeichnung Gymnastiksaal,79 die einige Aufseher dem Raum gegeben hätten.

Mit der Einrichtung einer ,Schutzhaftzentrale‘ beim Sächsischen Landeskriminalamt, über die die sächsischen Amtshauptmannschaften am 28. März 1933 schriftlich in Kenntnis gesetzt wurden, erfolgte der bereits skizzierte Versuch, einem offiziellen Anschein nach eine Gleichbehandlung der ,Schutzhäftlinge‘ in den zahlreichen frühen Haftstätten vorzutäuschen. Darüber hinaus waren die Häftlinge somit zumindest auf dem Papier vor Gewalt und Willkür geschützt. Dass diese Maßgaben in der Praxis weitestgehend keinerlei Anwendung fanden, werden die folgenden Schilderungen zeigen.

Unmittelbar nach der Ankunft - und das hatten die frühen Konzentrationslager trotz einiger unterschiedlicher Spezifika gemein - „machten die Angehörigen der Wachmannschaften deutlich, wie sie sich die Erziehung der Schutzhäftlinge vorstellten: mit Strafandrohungen, Gewalt und Misshandlungen." 80 Das erinnert an den sogenannten Willkomm, ein brutales Ritual, das im Zuchthaus Schloss Osterstein bereits 150 Jahre zuvor gepflegt wurde. Dieses Hemmzeichen fand auch anderthalb Jahrhunderte später seine Anwendung an gleicher Stelle. Gewaltsam fand eine deutliche Diffe-

73 Thies, Prozess (wie Anm. 31), S. 9.

74 Carina Baganz, Zwickau (Schloss Osterstein), in: Wolfgang Benz/Barbara Distel (Hg.), Der Ort des Terrors. Frühe Lager, Dachau, Emslandlager (Geschichte der nationalsozialistischen Konzentrationslager 2), München 2005, S. 227-230, hier S. 228; vgl. auch Thies, Prozess (wie Anm. 31), S. 4.

75 Baganz, Schloss Osterstein (wie Anm. 74), S. 228.

76 Vgl. ebd., S. $228 \mathrm{f}$.

77 Vgl. ebd., S. 228.

78 Ebd., S. 229.

79 Thies, Prozess (wie Anm. 31), S. 4.

80 Baganz, Volksgemeinschaft (wie Anm. 14), S. 155. 
renzierung zwischen der Welt außerhalb und innerhalb der Gefängnismauern statt und wurde ein deutliches Exempel der NS-Herrschaft statuiert. Unter dem Oberbegriff ,Erziehungsarbeit' verstandene Tritte und Schläge sowie Beschimpfungen menschenverachtender Art gehörten zu den ersten Augenblicken im Lager. ${ }^{81}$ So gibt die Anklageschrift des Osterstein-Prozesses von 1948 Einblick in einige Umgangsformen in Bezug auf Neuankömmlinge. Ein als Zeuge auftretender ehemaliger Häftling, auf den die Wärter nach eigenem Bekunden schon lange gewartet hatten, wurde unmittelbar nach seiner Ankunft durch Obrfeigen mißhandelt und mit dem Gummiknüppel geschlagen. ${ }^{82}$ Einem anderen Insassen wurden wenige Wochen später durch das Zufügen mehrerer Faustschläge ins Gesicht mebrere Zähne eingeschlagen und zersplittert, ${ }^{83}$ kaum dass er das Lager betreten hatte.

Die Bewachungsmannschaften bemühten sich in keiner Weise darum, ihre - so schreibt Sebastian Haffner - „feige und bleiche Fratze des leugnenden Mörders“ 84 auch nur im Ansatz zu verbergen, sondern zeigten sie bei der ersten sich bietenden Gelegenheit. Auch Rachegelüste konnten somit frühzeitig gestillt werden. Nicht zuletzt beweist das folgende Szenerie: Wir mußten uns in einer Reibe aufstellen, die Hände ausstrecken und mit den Worten: , Mit diesen Pfoten hast du auch Faschisten geschlagen', sausten die Hiebe auf die Hände herab. ${ }^{85}$

Die Bewältigung des Lageralltages betreffend, erfüllten die verschiedenen ,Begrüßungsrituale‘ eine weitere wesentliche, wenn vermutlich auch nicht durchweg beabsichtigte Funktion. Denn schon in dieser ersten Phase „lernten die Häftlinge, wie man den Anordnungen der Wachmannschaften nachkommen musste, um möglichst wenig aufzufallen. " 86 Und dennoch war jedem eingelieferten Häftling schnell klar, dass trotz solcher Bemühungen „eine Zeit voller Qualen, Schmerzen und Misshandlungen“87 bevorstand.

Oben, im Zandersaal haben diese vertierten Hunde mich so zugerichtet, 88 berichtete der Osterstein-Insasse Gottfried Reiter seinem Zellengenossen im Jahr 1933. Sinnbildlich für die dort verübten Gewaltverbrechen sei die Zeugenaussage des KPD-Mitgliedes Arthur Schmidt angeführt, der am 18. März 1933 verhaftet wurde. Nachdem er gezwungen wurde, sich völlig zu entkleiden, und auf die Frage nach angeblich versteckten Waffen keine Antwort geben konnte, wurde er zum Opfer einer nicht enden wollenden Abfolge von Verbrechen gegen seine Person: Und sofort fielen Bitterlich, Seidel und die anderen über mich her, warfen mich auf die am Boden liegende Matratze und schlugen wabllos auf mich ein. Ich habe ungefäbr 35 bis 40 Schläge gezählt, dann wurde ich bewußtlos. Als ich zu mir kam, begann die Fragerei von vorne. Und ebenso die Mißhandlungen. Das geschab noch mebrere Male in dieser Nacht. [...] Diese Vernehmung dauerte von nachts $12.30 \mathrm{Ubr}$ bis früh $6.00 \mathrm{Uhr}$. Kurz vor Schluß der Vernehmung haben mir Bitterlich und Seidel mit dem Gummiknüppel das Hakenkreuz [auf Brust und Rücken] eingeschlagen. [...] Am selben Tage nachmittags wurde ich wieder nach dem Zandersaal geholt und die Mißhandlungen begannen von neuem. Ich habe auch hier nichts ausgesagt. Darauf wurde ich an die Wand gestellt und Seidel

81 Vgl. ebd., S. 157.

82 Alle Zitate: Thies, Prozess (wie Anm. 31), S. 7.

83 Ebd.

84 Haffner, Geschichte eines Deutschen (wie Anm. 3), S. 125.

85 Thies, Prozess (wie Anm. 31), S. 10.

86 Baganz, Volksgemeinschaft (wie Anm. 14), S. 156.

87 Ebd., S. 159.

88 Thies, Prozess (wie Anm. 31), S. 4. 
schoß seine Pistole auf mich ab. Die Kugeln schlugen links und rechts neben meinem Kopf in die Wand. 89

Die verantwortlichen Aufseher handelten teils auf Befehl, teils aus eigenem Ermessen heraus. ${ }^{90}$ Welche Willkür dabei an den Tag gelegt wurde, belegt der Bericht des Zeugen Schmidt darüber, wie der Beteiligte an einer von einigen Wachleuten provozierten Kneipenschlägerei in der Zwickauer Innenstadt mitten in der Nacht nach Schloss Osterstein gebracht wurde. Politische Betätigungen, die eine Verfolgung nach NS-Richtlinien gerechtfertigt hätten, gab es nicht.

Außer dem Dachgeschoss bot auch der Keller des Zwickauer ,Schutzhaftlagers` ausreichend Platz für grausame Misshandlungen. In einer unterirdisch gelegenen 00-Zelle wurde in den letzten Apriltagen des Jahres 1933 ein führender KPD-Funktionär mit seinen Füßen an einem eingemauerten Ring am Fußboden ${ }^{91}$ angekettet und unter anderem durch den Einsatz von Gummiknüppeln bewusstlos geschlagen. Seinem Verwandtenbesuch sechs Wochen später musste er auf Druck der Wachen hin den gebrochenen Unterarm sowie das gebrochene Fußgelenk mit einem Sturz vom Dach erklären. ${ }^{92}$

Viele Zeugen berichteten 1948 von Torturen auf Schloss Osterstein; der ,Zandersaal', die Verliesen ähnelnden Zellen im Kellergeschoss oder andere Räumlichkeiten konnten zu jedem Zeitpunkt und ohne tatsächlichen Anlass zum Austragungsort der gewaltsamen Methoden der NS-Aufseher werden. Als abschließendes Beispiel und negativer Höhepunkt der menschenunwürdigen Zustände im Zwickauer, Schutzhaftlager' soll die Inhaftierung und Ermordung des KPD-Unterbezirkssekretärs Martin Hoop thematisiert werden. ${ }^{93}$ Am 2. Mai 1933 wurde Martin Hoop im Erfrischungsraum des Kaufhauses, Tietz' in Chemnitz verhaftet. In das Chemnitzer Polizeipräsidium gebracht, verschleppte man ihn von dort in das berüchtigte Konzentrationslager Schloß Osterstein. ${ }^{94}$ Dort musste Hoop nicht zuletzt aufgrund seiner Prominenz im Kreise der sächsischen KPD-Funktionäre schlimmste Folter über sich ergehen lassen. Die letzten Stunden Hoops lassen sich wohl wie folgt rekonstruieren: Hoop, der in einer Kellerzelle isoliert worden war, weigerte sich, den SS-Aufsehern gegenüber seinen Namen zuzugeben und durchlitt deswegen schwere Misshandlungen. Trotz verschlossener Zellentüren, so berichtet ein Zeuge, habe man hören können, wie die SS-Wachtrupps bei Hoop aus- und eingingen. ${ }^{95}$ Völlig erschöpft und unter Aufbringen seiner letzten Kräfte soll Hoop daraufhin darum gebeten haben, seinen Qualen ein Ende zu bereiten, indem man ihn erschieße; wenig später soll ein Schuss gefallen sein und tags darauf früb stand die Zellentür des Hoop offen und lediglich eine blutbefleckte Matratze ${ }^{96}$ sei zu sehen gewesen. In der Folge verbreitete das nationalsozialistische

93 Ebd.

93 Neben Hoops der Kommunistischen Partei in Osterstein umgekommen sein. Drei weitere Angehörige der KPD seien noch im Jahr 1933 an Folgen ihrer Misshandlungen gestorben und insgesamt zehn Angehörige von KPD und SPD hätten infolge der schweren Gewalttaten, die an ihnen verübt wurden, Suizid begangen. Das Wirken Martin Hoop's in Zwickau 1932/1933 (Beiträge zur Geschichte der Zwickauer Arbeiterbewegung 12), hrsg. vom Haus der Revolutionären Zwickauer Arbeiterbewegung, Zwickau 1984, S. 26.
Ebd. 
„Zwickauer Tageblatt“ auf Veranlassung der Wachmannschaften die Meldung, Hoop sei geflohen; dagegen sprechen nicht nur sein körperlich gebrochener Zustand, sondern auch die Aussage eines Zeugen, der SS-Wachposten Beetz [habe] im Garten der Anstalt ein großes Loch schaufeln lassen, in dem eine zusammen geschlagene Matratze mit weiteren Gegenständen vergraben wurde. ${ }^{97}$ Geklärt werden konnte eine konkrete Beteiligung an der Ermordung Martin Hoops bei keinem der 1948 angeklagten KZAufseher. Vieles spricht für die Theorie von den Tätern Bitterlich und Seidel, doch konnte eine direkte Verantwortung für Hoops Ermordung keinem der Angeklagten über alle Zweifel erhaben nachgewiesen werden. ${ }^{98}$

Die Belegschaft des Bewachungspersonals war im ,Schutzhaftlager' Schloss Osterstein von einigen Wechseln geprägt. Einer Bewachung durch die SS ab Anfang März folgte eine durch Polizisten ab Mitte März. Die SA bewachte das Lager ab Anfang April, bevor ab Mitte April erneut die SS einzog. ${ }^{99}$

Angelika Benz und Marija Vulesica stellen bezüglich der Täterforschung eine Vielzahl von „Einzel- und Kollektivbiografien“100 fest. Doch ähnlich wie Carina Baganz verweisen sie darauf, dass eine Generalisierung beispielsweise von Motivation und Ausführung der Bewachung nicht zielführend sei. ${ }^{101}$ Zwar könnten einige Merkmale angeführt werden, die durchaus auf einen großen Teil des Bewachungspersonals zutreffen, doch aus dem Fakt, dass viele Bewachungsmannschaften „aus arbeitslosen SA- und SS-Angehörigen [bestanden], denen außer ihrer Arbeitslosenunterstützung ein Tagesgeld von 1 Reichsmark, sowie freie Kost und Tabakwaren zur Verfügung gestellt wurden",102 kann nicht allumfassend auf die sozioökonomische Struktur der Bewacher in Zwickau geschlossen werden. Lediglich Parallelen können gezogen werden. Darüber hinaus beruhen die bekannten Informationen über die Aufseher der frühen Konzentrationslager einzig auf Stichproben und nicht etwa auf einem breiten und erschlossenen Aktenkorpus. ${ }^{103}$

Im Zeitraum vom März 1933 bis zum Ende des Januars 1934 waren Schätzungen zufolge circa 750 Personen ,Schutzhäftlinge' des Zwickauer Lagers. ${ }^{104}$ Dabei richtete sich das Vorgehen der Nationalsozialisten im Raum Zwickau vorwiegend „gegen Vertreter der organisierten Arbeiterbewegung" 105 von KPD, SPD, SAP und Gewerkschaften. Die Region liegt damit im Trend der Ereignisse des gesamten Reichsgebietes. In erster Linie betroffen waren die Anhänger und Funktionäre der Kommunistischen Partei. Sie markierten etwa die Hälfte der 1933/1934 einsitzenden politischen Gefangenen. Carina Baganz stellt fest, „dass zu Beginn des Lagers von März bis Mai 1933 fast ausschließlich Kommunisten, ab Juni 1933 verstärkt Sozialdemokraten zu verzeichnen sind."106 In diesen chronologischen Rahmen fügt sich auch die später vorgestellte

\footnotetext{
97 Ebd.

98 Darauf deuten sowohl Opfer- als auch Täteraussagen über mitgehörte Gespräche beziehungsweise das Entfernen eines Leichnams aus dem Schloss; vgl. ThiEs, Prozess (wie Anm. 31), S. 7, $10 \mathrm{f}$.

99 Vgl. Baganz, Volksgemeinschaft (wie Anm. 14), S. 148.

100 Angelika Benz/Marija Vulesica, Vorwort, in: Dies. (Hg.), Bewachung und Ausführung. Alltag der Täter in nationalsozialistischen Lagern (Geschichte der Konzentrationslager 1933-1945 14), Berlin 2011, S. 7-9, hier S. 7.

101 Vgl. ebd.; und Baganz, Volksgemeinschaft (wie Anm. 14), S. 150.

102 Baganz, Volksgemeinschaft (wie Anm. 14), S. 150.

103 Vgl. ebd.

104 Vgl. Baganz, Schloss Osterstein (wie Anm. 74), S. 228.

105 Baganz, Volksgemeinschaft (wie Anm. 14), S. 118.

106 Ebd., S. 120.
} 
Gruppe der Meeraner ,Schutzhäftlinge‘ ein. Das seit dem Frühsommer 1933 vermehrt auftretende Vorgehen gegen Sozialdemokraten scheint einher zu gehen mit dem Parteiverbot vom 23. Juni. In dessen Folge wurde die Verhängung von ,Schutzhaft ${ }^{`}$ über die entsprechenden Parteifunktionäre zunehmend von staatlicher Seite forciert. ${ }^{107}$

Die Mitglieder anderer Häftlingsgruppen, wie Juden, Homosexuelle oder Jehovas Zeugen, wurden „zu dieser Zeit nur vereinzelt verfolgt und in Lager überführt." 108 Bezüglich der zuerst genannten Gruppe sei aufgrund ihrer historischen Bedeutung auf den anonymen Bericht eines jüdischen Häftlings von Schloss Osterstein verwiesen: Nach der Machtergreifung der Nationalsozialisten wurde ich am 5. März 1933 in Aue aus meiner Wobnung verhaftet und in das dortige Polizeigewabrsam gebracht. Die Verhaftung nabmen Polizeibeamte obne Haftbefehl vor. Inzwischen füllte sich das Gefängnis in Aue mit Kommunisten, Sozialdemokraten und Juden, die alle aus dem Gebiet des Erzgebirges stammten. Einige Tage später wurde ein Teil der Inhaftierten - auch ich - in zwei Autobussen nach Schloß Osterstein in Zwickau gebracht. [...] Nach zwei Monaten Haft wurde ich aus Schloß Osterstein entlassen. Mir wurde nabegelegt, nichts über die dortigen Vorgänge zu erzäblen, da ich anderenfalls wieder in das $K Z$ käme. Mir gelang es dann, aus Deutschland nach der CSSR zu flüchten, und von dort gelangte ich nach Palästina. ${ }^{109}$

Die Erkenntnis, dass bereits unmittelbar nach der Erringung der Regierungsgewalt eine Vermischung von politisch und rassisch motiviertem Vorgehen gegen vermeintliche und tatsächliche ,Feinde' des NS-Regimes stattgefunden hat, lässt sich im Angesicht derartiger Berichte nicht leugnen. Zudem fügt sich beim Blick auf die Charakteristika der ,Schutzhäftlinge' von 1933 und 1934 ein weiterer Aspekt in unser Bild ein. Denn im Bestreben, „die politischen Gefangenen mit Kriminellen gleichzusetzen, dadurch zu diskriminieren und noch stärker zu tyrannisieren", 110 wurde das breite Spektrum der Inhaftierten noch im Jahr 1933 ergänzt durch kriminelle Personen im traditionellen Verständnis. Noch bevor viele frühe Konzentrationslager (wieder) zu Zuchthäusern und ähnlichen Einrichtungen umfunktioniert wurden - wie beispielsweise Schloss Osterstein - muss demnach auch eine bewusst gesteuerte Vermischung dieser Haftmotive diagnostiziert werden. Somit seien „Vertreter fast aller späteren Häftlingsgruppen bereits in den frühen Konzentrationslagern zu finden " 111 gewesen.

Am 14. Juli 1933 wandte sich der Stadtrat des sächsischen Meerane an die Leitung des Konzentrationslagers Schloss Osterstein im wenige Kilometer entfernten Zwickau. Sich auf ein tags zuvor erhaltenes Schreiben vom stellvertretenden Kreiskommissar namens Dr. Müller berufend, bat der Stadtrat höflich um die Entlassung von 14 Gefangenen aus der Haftanstalt. Grundlage für diese Bitte und ihre Erfüllung war eine Verfügung aus dem Ministerium des Innern, in deren Rahmen am 11. Juli 1933 die Entlassung der SPD-Funktionäre forciert worden war. Nach dem Verbot der Sozialdemokratischen Partei am 23. Juni 1933 stellten ihre Mitglieder und Funktionäre für den nationalsozialistischen Staat keine legale Gefahr mehr da. Lag gegen die Betreffenden kein besonderer Anla $\beta^{112}$ zur weiteren Inhaftierung vor, wurde ihre Entlassung in vielen Fällen genehmigt.

Von zwölf der vierzehn erwähnten und freigelassenen Insassen des KZ Schloss Osterstein konnten im Staatsarchiv Chemnitz die entsprechenden Dokumente zu Bio-

107 Vgl. u. a. StA Chemnitz, 30063, Nr. 57.

108 Baganz, Volksgemeinschaft (wie Anm. 14), S. 118.

109 Adolf Diamant, Zur Chronik der Juden in Zwickau, Frankfurt a. M. 1971, S. 45.

110 Baganz, Volksgemeinschaft (wie Anm. 14), S. $61 \mathrm{f}$.

111 Ebd., S. 61.

112 StA Chemnitz, 30063, Nr. 65. 
grafie, Inhaftierung und Nachüberwachung gefunden werden. ${ }^{113}$ Ihre großen Gemeinsamkeiten bestehen einerseits in der Herkunft aus Meerane und andererseits in der Inhaftierungsdauer vom 25. Juni bis zum 15. Juli 1933. Das bearbeitete Aktenkorpus bietet daher eine im Rahmen dieser Arbeit günstige Gelegenheit, am Exempel der Meeraner Sozialdemokraten und Kommunisten die Vorgänge im Rahmen der ,Schutzhaft im Zwickauer Raum zu schildern.

$\mathrm{Zu}$ den Charakteristika der im Mittelpunkt stehenden Personen: Die OstersteinHäftlinge des Sommers 1933 waren ausschließlich Männer im durchschnittlichen Alter von 45,5 Jahren. Dieser Wert ergibt sich aus elf bekannten Geburtsdaten der Gruppe. Der älteste Häftling war zum Zeitpunkt der Inhaftierung 63, der jüngste 30 Jahre alt. Zehn der Insassen waren zuvor Mitglieder der SPD, zwei der KPD gewesen. Das überrascht, wurde die Verhaftung aller zwölf Personen am 25. Juni doch folgendermaßen gerechtfertigt: Aufgrund der Anordnung des gebeimen Staatspolizeiamtes [...] vom 23. Juni 1933 alle fübrenden SPD-Funktionäre sofort in Schutzhaft zu nebmen, wurden beute Sonntag, den 25. Juni 1933, nachmittags 3 Ubr nachfolgend Genannte dem Konzentrationslager Zwickau, Schloß Osterstein, zugefübrt. ${ }^{114}$ Dennoch finden sich unter den Verhafteten zwei Anhänger der Kommunistischen Partei, die mit Verweis auf dieselbe Anordnung nach Osterstein überführt wurden. Vermutlich zeigt sich unter anderem an dieser Stelle entweder das Scheitern von Regulierungsversuchen durch Reichs- und Landesregierung und/oder ein konkretes Beispiel der Willkür, die im Umgang mit politisch Unliebsamen an den Tag gelegt wurde.

Fünf der zwölf Männer waren bis zu ihrer Verhaftung in der Textilbranche tätig. Darüber hinaus finden sich ein Elektriker, ein Krankenkassenkassierer sowie ein Gewerkschaftsbeamter. Über den Beruf von vier Gefangenen konnte keine Angabe gefunden werden. Ein weiterer bemerkenswerter statistischer Wert lässt einen Aufschluss darüber zu, inwiefern die Maßnahme ,Schutzhaft' tatsächliche Wirkung zeigte: Bei sechs der zwölf Personen wurden offene und geheime Nachüberwachung nach durchschnittlich sieben Jahren eingestellt mit dem sinngemäßen Hinweis, der Überwachte gliedere sich inzwischen in die ,Volksgemeinschaft' ein und sei keine Gefahr mehr für den Staat. Doch inwiefern setzte die Institution Osterstein die NS-Herrschaft im Raum Zwickau konkret durch?

Mit der Entlassung aus dem ,Schutzhaftlager' Schloss Osterstein am 15. Juli 1933 waren die Repressionen für die zehn Sozialdemokraten und zwei Kommunisten aus Meerane nicht beendet. Vielmehr standen die zwölf Personen weiterhin im Fokus der vor Ort verantwortlichen Nationalsozialisten. So wurde die Freilassung aus dem Lager an strenge Auflagen geknüpft, die die betroffenen Personen penibel einzuhalten hatten. Dazu gehörte erstens die eingangs zitierte Erklärung zu Loyalität gegenüber dem NS-Regime, zur Unterlassung staatsfeindlicher Agitation und zum Verzicht auf gegebenenfalls entstandene Entschädigungsansprüche, etwa durch erlittene körperliche Schäden im KZ. ${ }^{115}$ Zweitens - und das führte neben dem Verlust des Arbeitsplatzes häufig zu einer schlechten finanziellen Lage - wurde den ehemaligen Insassen die Zeit im Schloss Osterstein mit zwei Reichsmark pro Tag in Rechnung gestellt. Dies regelte eine Verfügung des Innenministeriums vom April 1933. Für den Zeitraum vom 25. Juni bis zum 15. Juli 1933 ergibt sich für die zwölf Meeraner Häftlinge eine Summe von je 40 Reichsmark. Welche Lücken diese Kosten in die Brieftaschen der Betroffenen riss, belegt folgende Bitte um Erlass der ,Schutzhaftkosten' an den Stadtrat Meerane vom August 1933: Bis zum 24. 6. 33 war ich weder polizeilich noch Gerichtlich bestraft.

113 StA Chemnitz, 30063.

114 Ebd., Nr. 57.

115 Vgl. u. a. ebd., Nr. 36. 
Durch die Inschutzhaftnabme meiner Person wurde[n] mir [...] die Papiere zur Entlassung aus den [sic!] Arbeitsverbältnis zugestellt. Durch die erfolgte Arbeitslosigkeit sind meine finanziellen $u$. familiären Verbältnisse erbeblich verschlechtert, damit kann ich meinen Verpflichtungen als Haushaltsvorstand nur schwer nachkommen, denn von 12,60 M Unterstützung 4 Personen zu erbalten $u$. ausserdem noch die mir auferlegten Kosten für meine Schutzhaft in Höhe von 40,00 M zu tragen, dazu bin ich nicht in der Lage. ${ }^{116}$

Eine später erfolgte Antwort seitens des Stadtrates deutet darauf hin, dass dem Betroffenen eine regelmäßige Ratenzahlung angeboten wurde. Dieses Vorgehen, das sich letztlich pragmatisch damit begründet, dass eine Zahlung anderweitig nicht zu erwarten gewesen wäre, findet sich auch im Umgang mit anderen ehemaligen Osterstein-Häftlingen, die ähnliche Bitten formulierten.

Eine dritte repressive Methode nach der ,Inschutzhaftnahme‘ stellte die Auferlegung strenger Meldepflichten dar: Es war den ehemaligen Häftlingen auferlegt worden, sich täglich einmal nachm. 2 Ubr auf der Polizeiwache zu melden. Eine Befreiung war mindestens 48 Stunden, ein Urlaubsgesuch eine Woche vorher einzureichen. Zwischen 22 und 5 Uhr waren sie zum Aufenthalt in der Wohnung verpflichtet. Ferner war das Verlassen des Stadtgebietes [...] obne besondere Genebmigung verboten. Verstöße wurden geahndet mit sofortige[r] Inschutzhaftnabme. ${ }^{117}$

Die Anwendung dieser weiteren Maßnahme zeigt klar und deutlich das Bestreben, die als politische Gegner Identifizierten auch nach Entlassung aus der ,Schutzhaft ${ }^{`}$ zu kontrollieren. Das Damoklesschwert einer erneuten Inhaftierung schwebte stets über ihrem gesamten Verhalten in der Öffentlichkeit und im Privaten. Die sogenannte Erziehung zur Volksgemeinschaft fand ihr Ende damit nicht etwa beim Verlassen der Haftanstalt, sondern auch durch den Zugriff durch offene und geheime Bespitzelung im eigenen Ort, einer vierten Maßnahme. Durchschnittlich sieben Jahre dauerte die Nachüberwachung im Falle der zwölf Ex-Häftlinge an, teilweise sogar bis in das Jahr 1941.118 Dass die Nachüberwachung in vielen Fällen nach mehreren Jahren aufgehoben wurde, lässt sich immer wieder auf die Feststellung zurückführen, die überwachte Person habe sich in ihrer politischen Gesinnung gewandelt. Zwei dieser Fälle seien nun genauer beschrieben.

Noch im November 1940 wurde ein ehemaliger Osterstein-Häftling vom Meeraner Oberbürgermeister als unbelebrbar und noch nicht in die Volksgemeinschaft eingefügt ${ }^{119}$ eingeschätzt, die Überwachung folglich aufrechterhalten. Bereits im April 1941, also lediglich fünf Monate später, wurde die Nachüberwachung unter Berufung auf folgendes Schreiben zugunsten des überwachten ehemaligen Sozialdemokraten jedoch aufgehoben: Nach seiner Entlassung aus der Schutzhaft war er zurückbaltend und verschlossen. Seit längerer Zeit gehört aber nun sein Sohn dem Jungvolk an, woraus zu schließen ist, daß er mit der politischen Meinung seines Sobnes einverstanden

116 Ebd., Nr. 38.

117 Alle Zitate: ebd., Nr. 36. Solche Auflagebestimmungen finden sich, wenn auch mit teils variierenden Ausgestaltungen, in nahezu allen gesichteten Akten.

118 Im Fall eines als eingefleischte[n] Marxist[en] geltenden ehemaligen Sozialdemokraten blieb die Aufrechterhaltung der Nachüberwachung über den Februar 1941 hinaus bestehen, kann danach jedoch nicht mehr nachverfolgt werden. Sie prinzipiell bis Kriegsende beziehungsweise dem (unbekannten) Todesdatum anzunehmen, scheint jedoch nicht durchweg begründet zu sein, da auch andere ,Unverbesserliche` durchaus von Überwachungsmaßnahmen befreit wurden. Vgl. dazu auch StA Chemnitz, 30063, Nr. 36.

119 Beide Zitate: ebd. 
ist. Bei Sammlungen schließt er sich nie aus und gibt seinen Verbältnissen entsprechend auch reichlich. In Anbetracht dessen, daß [er] zur Webrmacht einberufen ist und sich auch vorher bemüht hat, sich in die Volksgemeinschaft einzufügen, bitte ich um Entschließung wegen Aufhebung der Nachüberwachung. ${ }^{120}$

Ist dieser Gesinnungswandel in erster Linie durch seine Plötzlichkeit charakterisiert, so ist es der folgende durch sein Ausmaß. Erneut erfolgt ein Bezug auf den Lebensweg eines SPD-Mitgliedes, das bis zum Januar 1933 sowohl das ,Reichsbanner sowie die ,Eiserne Front' unterstützte und damit die NS-Bewegung klar und deutlich bekämpfte. Aufgehoben wurde seine Nachüberwachung im Juni 1940. Die dieser Entscheidung zugrunde liegende Erklärung des Polizeihauptwachtmeisters in Meerane lautete: [Der ehemalige Sozialdemokrat] ist Mitglied der DAF und gebört dem Feldkameradenbund (Pionierabteilung) an. [...] Es hat den Anschein, daß er sich in politischer Beziebung geändert hat. Eine gegen den Staat gerichtete politische Tätigkeit ist jedenfalls von ihm nicht bekannt geworden. Seine Tochter [...] ist Angebörige der HitlerJugend. Anläßlich der Volksabstimmung am 10.4.1938 hatte [...] [er] fast sämtliche Fenster seiner im Erdgeschoß gelegenen Wohnung mit Hakenkreuz-Fähnchen und Blumen geschmückt. Außerdem hatte er an zwei Fenstern Wablaufforderungen angebracht. ${ }^{121}$

Beide geschilderten Schicksale werfen ebenso Fragen auf wie andere festgestellte Änderungen der politischen Einstellung bei ehemaligen ,Schutzhäftlingen'. Im Vordergrund stehen sicherlich die nach der Authentizität der durch die jeweiligen NS-Verantwortlichen diagnostizierten Veränderungen. Die Wahrhaftigkeit derartiger Gesinnungswechsel einmal angenommen, würde sich wiederum die Frage nach den direkten und indirekten Ursachen dafür auftun.

\section{Die Aufarbeitung - Der Osterstein-Prozess von 1948}

Die Jahre 1933/1934 bedeuteten für schätzungsweise 750 Menschen eine Inhaftierung im Zwickauer ,Schutzhaftlager' Schloss Osterstein. Physische und psychische Folter gehörten für die Häftlinge zum Leben hinter den Mauern dieses frühen Konzentrationslagers. Im Jahr 1948 wurden die dortigen Misshandlungen, die an der Tagesordnung waren, ${ }^{122}$ in einem Gerichtsprozess aufgearbeitet. Gegen vier ehemalige Wachpersonen, die aufgegriffen werden konnten, wurde dabei Anklage erhoben. Namentlich mussten sich Kurt Herbert Bitterlich, Wilhelm Josef Spindler, Willy Karl Rothkirch und Kurt Paul Kupfer für ihre Gewalttaten verantworten. Der als, Volksankläger' auftretende Franz Thies hat die Hauptvorwürfe und wesentlichen Ermittlungsergebnisse dieses Prozesses in einer dazu erschienenen Broschüre aufgeführt. Dass seine Darstellung einer politisch begründeten Tendenz unterliegt, soll nicht verschwiegen werden. Dennoch eignet sich die Thies-Schrift für die Beleuchtung der Konsequenzen, die die Vorkommnisse auf Schloss Osterstein zumindest für die festgenommenen KZ-Aufseher hatten. Zuerst einmal muss konstatiert werden, dass den geforderten Strafen im Urteil vom 17. April 1948 nicht nachgekommen wurde. Ein Vergleich von geforderter und tatsächlich verhängter Strafe ergibt folgendes Bild:123

120 Ebd.

121 Ebd.

122 Thies, Prozess (wie Anm. 31), S. 8.

123 Das volle Urteil ist weder bei Thies noch in einer herangezogenen Sammlung von Urteilen gegen NS-Verbrecher zu finden. Vielmehr erfolgt folgender Hinweis: „Von den in diesem Verfahren ergangenen Entscheidungen wurde in den einschlägigen Archi- 
Bitterlich, Kurt Herbert

Forderung: Verurteilung zum Tode, dauernder Ehrverlust

Urteil: lebenslange Haft ${ }^{124}$

Spindler, Wilhelm Josef

Forderung: 15 Jahre Zuchthaus, dauernder Ehrverlust

Urteil: 15 Jahre Zuchthaus

Rothkirch, Willy Karl und Kupfer, Kurt Paul

Forderung: 8 Jahre Zuchthaus, Ehrverlust für 10 Jahre

Urteil: 6 Jahre Zuchthaus. ${ }^{125}$

Neben den hier genannten NS-Straftätern waren viele weitere, auch namentlich bekannte Aufseher an den Gräueltaten im Schloss Osterstein beteiligt. Ihrer konnte nach dem Krieg jedoch offenbar niemand habhaft werden. Eine Ironie der Geschichte ist es, dass die 1948 Verurteilten zumindest ihre Untersuchungshaft an dem Ort verbrachten, dem sie fünfzehn Jahre zuvor noch den Charakter einer Folterstätte verliehen hatten.

\section{Fazit}

Der Osterstein-Prozess findet seine Erwähnung nicht nur der Vollständigkeit halber, sondern weil er darüber hinaus Aufschluss über eine der verschiedenen Funktionen der Institution Schloss Osterstein gibt. Denn vor einer Auseinandersetzung mit systematischen Aspekten muss auf die Bedeutung simpler Rachegelüste hingewiesen werden, die durch weite Teile des Wachpersonals in den frühen Konzentrationslagern immer wieder ausgelebt wurden. Im Zusammenhang mit den verschiedenen ,Schutzhafterlassen' des Jahres 1933 wurde auf den vermutlich besonders hohen Grad der Gewaltbereitschaft vor allem in den sächsischen Lagern hingewiesen. ${ }^{126}$ Parallel dazu begann ein Einschwören eines potenziellen Täterkreises auf später noch durchzuführende Aktionen gegenüber der jüdischen Bevölkerung, politisch Unliebsamen, Homosexuellen, Jehovas Zeugen und anderen durch die Nationalsozialisten verfolgten Personengruppen. Gewissermaßen führte die Allgegenwärtigkeit von Gewalt, die Deutschland im Frühjahr 1933 prägte, zu einem deutlichen Sinken der Hemmschwelle. Denn trotz der auch schon vor dem Januar 1933 ausgetragenen ,Kämpfe' erreichte das Vorgehen gegen vermeintliche und tatsächliche NS-Gegner mit der Machtübernahme und dem Reichstagsbrand eine ganz neue Qualität. Regulierungsversuche von staatlicher Seite erscheinen unter Berücksichtigung dieser Umstände als Farce, ein wirkliches Interesse an einer menschenwürdigen Gleichbehandlung der ,Schutzhäftlinge“ als nicht glaubwürdig.

Im Rückblick auf die ,späten' Konzentrationslager, mit denen vor allem Auschwitz und Buchenwald assoziiert werden, ist die immer wieder geltend gemachte Unkennt-

ven nur ein Bruchteil angetroffen. Auch das dieses Verfahren abschliessende rechtskräftige Urteil des LG Zwickau vom 17.4.1948 fehlt.“; ChristiaAn Frederik RÜTER/L. HekelaAr Gombert/Dick W. De Mildt, DDR-Justiz und NS-Verbrechen. Sammlung Ostdeutscher Strafurteile wegen Nationalsozialistischer Tötungsverbrechen, Bd. 11: Die Verfahren Nr. 1610-1692 des Jahres 1948, München/Amsterdam 2008, S. 287.

124 Ein Antrag auf Rehabilitierung wurde am 16. November 1993 abgelehnt.

125 Eine Feststellung der gesprochenen Urteile findet sich lediglich über die Onlinepräsenz der eben zitierten Sammlung: RüTER/Gombert/De Mildt, DDR-Justiz und NS-Verbrechen (wie Anm. 123), Verfahren Lfd.Nr.1640, http://www1.jur.uva.nl/junsv/ddr/ files/ddr1640.htm [Zugriff: 28. März 2016].

126 Vgl. Baganz, Volksgemeinschaft (wie Anm. 14), S. 72. 
nis der Bevölkerung bis heute ein umstrittener Punkt der Forschung. Was die frühen Lager betrifft, so kann Unwissenheit für die meisten Bürgerinnen und Bürger wohl nicht in Anspruch genommen werden. Zu weite Kreise zogen die Nachrichten über verschleppte Familienväter oder beispielsweise (ehemalige) Stadtverordnete. Vielmehr noch: Seitens des NS-Regimes sowie der vor Ort Verantwortlichen bestand gar ein Interesse an der Verbreitung derartiger Nachrichten. ${ }^{127}$ Neben aggressiven Ankündigungen, wie mit Kommunisten und Sozialdemokraten umgegangen werde, ${ }^{128}$ druckten auch Zeitungen immer wieder Schilderungen der Verhaftungen ab. Ein Artikel aus dem „Zwickauer Tageblatt“ vom 11. März verriet den Leserinnen und Lesern Folgendes: Es erfolgten auch in Zwickau noch neue Zwangsstellungen [...], besonders von marxistischen Stadtverordneten beziehungsweise Landtagsabgeordneten, auch sind zum Teil zur Entlassung gekommene wieder zurückgeholt worden. ${ }^{129}$

Was die Haftumstände anbelangt, so wurde jedoch schlichtweg gelogen. Als zuvorkommend und interessiert am Wohlbefinden der Insassen wurde etwa das Verhalten der Osterstein-Aufseher im selben Artikel beschrieben. ${ }^{130}$ Trotz dieser zynischen Agitation verrieten die „Schreie der gefolterten Häftlinge im berüchtigten Nordgebäude“131, was hinter den Schlossmauern tatsächlich geschah und in seiner Lautstärke den Anwohnern immer wieder den Schlaf raubte. Sebastian Haffner umschreibt die Funktion der vor dem Hintergrund der zahlreichen ,Inschutzhaftnahmen' gesteuerten Propaganda treffend: „Selbstverständlich bezweckte das nicht, die Greuel wirklich geheim zu halten. Dann hätten sie ja ihren Zweck, allgemein Furcht, Schrecken und Unterwerfung hervorzubringen, nicht erreichen können. Vielmehr sollte die Terrorwirkung gerade durch das Geheimnis gesteigert werden und durch die Gefahr, die darin lag auch nur darüber zu reden. Die offene Darstellung dessen, was in den SA-Kellern und Konzentrationslagern geschah [...], hätte möglicherweise selbst in Deutschland verzweifelte Gegenwehr hervorgerufen. Die heimlich herumgeflüsterten schaudervollen Geschichten [...] brachen viel sicherer jedes Rückgrat." 132

Den machtpolitischen Erfolg kann man dieser Doppelstrategie aus offenem Drohen mit Verschleppung und der Bewahrung einiger Geheimnisse über die konkreten Haftumstände nicht absprechen. ${ }^{133} \mathrm{Zu}$ umfassend fielen der im Frühjahr 1933 stattfindende Kollaps der Rechtsstaatlichkeit und die wie auch immer motivierte Gefolgschaft gegenüber Hitler in der Folge aus.

Eine durchaus naheliegende, aber nicht zu vernachlässigende Aufgabe des Zwickauer ,Schutzhaftlagers' lag in der pünktlichen Ausschaltung von Oppositionellen vor der Reichstagswahl am 5. März 1933. Im Juni desselben Jahres wiederholte sich ein solches Bestreben nach dem Verbot der Sozialdemokratischen Partei. Mag der Ärger über das Verfehlen einer absoluten Mehrheit trotz zweistelliger Zugewinne auf Seiten der NSDAP noch so groß gewesen sein, müssen die Verluste von SPD und insbesondere KPD ihre Erwähnung finden. Die Demobilisierung vieler verantwortlicher Parteifunktionäre, in der frühen Phase ja vor allem bei der Kommunistischen Partei, schlug sich im Wahlergebnis definitiv nieder. Die Verfolgung und massenhafte Inhaftierung von Sozialdemokraten gewann dann ab Juni zunehmend Dynamik. Neben dem Verschwinden wichtiger Würdenträger wurden die - nach und nach in den Unter-

127 Ebd., S. 65.

128 Siehe u. a. Wilhelm Fricks Ankündigung vom 8. Dezember 1932.

129 Peschke, Naziterror (wie Anm. 18), S. 24.

130 Vgl. ebd., S. 38.

131 Ebd.

132 Haffner, Geschichte eines Deutschen (wie Anm. 3), S. 126.

133 Vgl. BagAnz, Volksgemeinschaft (wie Anm. 14), S. 64. 
grund gedrängten - betroffenen Parteistrukturen durch einen zusätzlichen Aspekt nachhaltig geschwächt. Denn selbst eine Entlassung aus der Haft änderte nichts daran, dass die Betroffenen für die politische Agitation gegen das NS-Regime nicht mehr infrage kamen, ohne ihr Leben und das ihrer Angehörigen zu riskieren. Die Schicksale der nachüberwachten Sozialdemokraten und Kommunisten aus Meerane belegen das. Das ständige Damoklesschwert einer erneuten Inhaftierung, von deren unmenschlichen Bedingungen die entlassenen Häftlinge ja auch berichteten, führte nach und nach zu einer Zermürbung oppositioneller Bemühungen.

Die Absonderung von Parteimitgliedern von SPD, KPD und auch SAP und ihre später einsetzende Wiedereingliederung in die sogenannte Volksgemeinschaft erfüllte darüber hinaus eine weitere Doppelfunktion: Einerseits wurde mit ihrer Entfernung aus ihrem gewohnten Umfeld jede Form der politischen Betätigung und Werbung unterbunden. Andererseits diagnostizieren wir nach der Entlassung aus der ,Schutzhaft ${ }^{\prime}$ in nicht wenigen Fällen sogar einen politischen Gesinnungswandel. Gegebenenfalls konnte dadurch mittels ehemaliger politischer Gegner sogar ein Vorbildcharakter für andere Bürgerinnen und Bürger geschaffen werden. Blieben einige OstersteinHäftlinge nach ihrer Entlassung auch offen überzeugte Sozialdemokraten oder Kommunisten und hatten mit den entsprechenden Konsequenzen zu leben, finden sich in den entsprechenden Akten auch deutliche Gegenbeispiele. So wird ein ehemaliger Sozialdemokrat aus Meerane im Januar 1939 geschildert als gute[r] und willige[r] Mensch, der unter seinen Arbeitskameraden vorbildlich für die NSDAP und für die Reichsregierung eingetreten ist und auch in Zukunft eintreten wird. ${ }^{134}$ Gewiss lassen diese Forschungsergebnisse keinen durchweg verlässlichen Schluss darüber zu, inwiefern sich politische Gesinnungen bei ehemaligen KZ-Häftlingen tatsächlich gewandelt haben oder ob es sich hierbei nur um ein Schutzverhalten für sich und die eigene Familie handelte. Hierfür müssten andere Quellentypen herangezogen werden, vorrangig persönliche Dokumente wie beispielsweise Tagebücher. Doch selbst eine nur nach außen gezeigte Zustimmung für den NS-Staat oder sogar seine Unterstützung mobilisiert andere Mitglieder der Gesellschaft dazu, sich ähnlich zu verhalten.

Das Ziel der frühen Konzentrationslager der Jahre 1933/1934 und damit auch des ,Schutzhaftlagers' Schloss Osterstein sieht Carina Baganz in der „umfassende[n] Ausschaltung des politischen Gegners und [...] [der] damit einhergehende[n] Stabilisierung des nationalsozialistischen Systems." ${ }^{135}$ Dass dieses Ansinnen auf verschiedenen Wegen erreicht wurde, zeigen die Ergebnisse der vorliegenden Studie.

Schloss Osterstein kam damit eine wesentliche Rolle beim Prozess der nationalsozialistischen Machtkonsolidierung im Raum Zwickau zu. Das mag eine gewagt erscheinende Schlussfolgerung sein im Angesicht eines Aktenkorpus von verhältnismäßig eher kleinerem Umfang. Doch besteht kein plausibler Grund dafür, in anderen Gebieten des Zwickauer Umlandes deutlich andere Verhältnisse anzunehmen. Norbert Peschkes mehrfach zitierte Studie gibt Aufschluss über ähnlich wie in Meerane stattgefundene Verhaftungen unter anderem in Adorf, Markneukirchen, Oelsnitz i. V., Plauen, Reichenbach, Werdau, Leubnitz und Auerbach. ${ }^{136}$ Das dürfte bei weitem keine erschöpfende Aufzählung sein. Vielmehr ist ein Ausgreifen der Institution Osterstein auf das gesamte Zwickauer Umland anzunehmen, denn schließlich durchlitten circa 750 Häftlinge dieses Lager zwischen März 1933 und Januar 1934. Darüber hinaus sprechen die Berichte aus anderen frühen Lagern und auch der Erfolg der ausgiebigen nationalsozialistischen Machterringung während des Jahres 1933 für die

134 StA Chemnitz, 30063, Nr. 83.

135 Baganz, Volksgemeinschaft (wie Anm. 14), S. 64.

136 Vgl. Peschke, Naziterror (wie Anm. 18), S. 47. 
große Rolle der frühen Lager, zu denen Schloss Osterstein gehörte. Allein die seit Sommer 1933 zunehmend gewährten Entlassungen aus der ,Schutzhaft ${ }^{\star}$ waren weniger auf Platzmangel zurückzuführen als vielmehr auf das schiere Gelingen der durch die Nationalsozialisten ausgerufenen ,Revolution'. Ähnlich formuliert Carina Baganz diese Erkenntnis: „Die Konzentrationslager der Jahre 1933 und 1934 waren die Basis für die schnelle Machtetablierung des Nationalsozialismus. Durch ihre sofortige Einrichtung ab März 1933 und die damit einhergehenden gewalttätigen Maßnahmen gegen die politischen Gegner war die nationalsozialistische Herrschaft im Sommer 1933 bereits so weit gefestigt, dass Adolf Hitler am 6. Juli 1933 den ,Abschluss der Revolution“ verkünden konnte [...].“137

Aus den präsentierten Forschungsergebnissen ergeben sich naturgemäß Desiderate, insbesondere bei einer Arbeit geringeren Umfangs. Zuerst sei der Aspekt aufgeworfen, dass bis heute keine umfassende Auflistung der 1933/1934 in Osterstein inhaftierten Personen existiert. Im Rahmen dieser Arbeit wäre eine solche Aufstellung auch nicht von zielführendem Charakter gewesen, da nahezu alle Akten aus dem Staatsarchiv Chemnitz nach wie vor mit einer Schutzfrist versehen sind. Darüber hinaus kann bei den in der Literatur verwendeten Namen nicht ausgeschlossen werden, dass aufgrund eben dieser Fristen die Klarnamen der Opfer abgeändert wurden. Der Gefahr einer Dopplung müsste bei einem entsprechenden Projekt sicherlich Rechnung getragen werden. Die Möglichkeiten für eine eingehendere Studie zu Schloss Osterstein bestehen im Angesicht des Ausmaßes der archivierten Akten durchaus. Das birgt das Potenzial für eine chronologische Osterstein-Studie ebenso in sich wie eine deutlich umfangreichere Arbeit zum Aufstieg der NSDAP in Zwickau und Umgebung bis hin zum Kriegsende, die Institution Schloss Osterstein stets einbezogen.

Des Weiteren lässt die Beschäftigung mit dem Osterstein-Prozess von 1948 wohl deutlich mehr Fragen offen, als sie Antworten geben kann, zumindest unter dem $\mathrm{Zu}$ griff auf die bekannten Dokumente. Franz Thies' Bericht leidet trotz seines hohen Informationsgehaltes sowohl an seinem recht unstrukturierten Charakter wie auch an seiner stark ausgeprägten Moralisierung und daher mangelnden wissenschaftlichen Distanz. Bleibt es die entsprechenden Verfahrensakten betreffend jedoch bei den erwähnten Bruchstücken, wird ein entsprechendes Vorhaben der weiteren Aufarbeitung und Erforschung wohl stets mit großen Problemen konfrontiert sein. Eine Einreihung in die bisherige Täterforschung, die unter anderem durch den Bezug auf Benz und Vulesica angedeutet wurde, könnte im Rahmen einer Studie zum Osterstein-Prozess auch durchgeführt werden.

Ein weiterer Forschungsschwerpunkt, der fortan deutlicher im Mittelpunkt stehen könnte/sollte, ist der von der These der kollektiven Unwissenheit über die Ereignisse in den auf deutschem Territorium befindlichen Lager. Unter anderem anhand der Erkenntnisse dieser Arbeit, aber auch bezugnehmend auf Forschungsergebnisse einer Jenaer Forschergruppe um Rüdiger Stutz, Marc Batuschka und Katrin Fügner muss konstatiert werden, dass die deutsche Zivilbevölkerung keinesfalls unwissend darüber war, „was ,die Nazis‘ zwischen 1933 und 1945 in den Lagern taten.“138 Verwiesen sei auf die von Norbert Peschke angeführten Schreie der Insassen, welche über die Schlossmauern hinaus hörbar gewesen seien, ${ }^{139}$ aber auch auf die Zwangsarbeitskräfte, welche „direkt neben den regulären Arbeitern" 140 eingesetzt worden sind. Ihre Aus-

137 Baganz, Volksgemeinschaft (wie Anm. 14), S. 65.

138 Sebastian HaAk, Die Zwangsarbeit gleich nebenan, in: Neues Deutschland, 10. April 2015, S. 3.

139 Vgl. Thies, Prozess (wie Anm. 31), S. 38.

140 HaAk, Zwangsarbeit (wie Anm. 138), S. 3. 
beutung sei in Deutschland allgegenwärtig gewesen; Zahlen liegen u. a. für Jena, Suhl, Altenburg und Gotha vor. ${ }^{141}$ Deswegen, so schlussfolgert Sebastian Haak, müssten die meisten „in Deutschland in den 1940er Jahren zumindest eine Vorstellung davon gehabt haben, was in NS-Lagern passierte; wenngleich die Details des industriellen Tötens in den Konzentrationslagern im Osten Europas vielleicht wirklich ein Geheimnis waren, das den meisten Deutschen erst nach Ende des Krieges offenbar wurde." 142 Ein wissenschaftlich fundiertes Aufräumen mit diesem über Jahrzehnte hinweg beschworenen Mythos sollte im Interesse aller seriösen Historikerinnen und Historiker sein, die über den Nationalsozialismus forschen.

Letztlich stellt auch der geschilderte radikale politische Gesinnungswandel von ehemaligen KZ-Häftlingen ein noch nicht ausreichend erforschtes Gebiet dar. Sowohl die Motive als auch die Echtheit dieser Wandlungsprozesse stellen uns vor die Aufgabe, entsprechende Quellentypen gegebenenfalls zugänglich zu machen und aufzuarbeiten.

Fest steht, dass die frühen Konzentrationslager einen ersten Schritt auf dem Weg zur Errichtung und Etablierung eines viel größeren Systems von Arbeits- und Vernichtungslagern markierten, "die bis zum heutigen Tage als Synonym für die NS-Herrschaft angesehen werden. "143 Diese Studie zeigt jedoch, dass eine Reduzierung des Nationalsozialismus auf die späteren Lager nicht nur historisch ungenau ist, sondern den Opfern der Frühphase des sogenannten Dritten Reiches nicht gerecht wird. ${ }^{144}$ Damit wird nicht etwa die Tatsache verharmlost, dass in späteren Lagern Millionen Tote zu verzeichnen waren, sondern vielmehr darauf verwiesen, dass die nationalsozialistische Diktatur schon sehr frühzeitig einen von Gewalt, Entrechtung und Menschenverachtung geprägten Weg beschritt.

141 Vgl. ebd. Bezüglich des Gebietes des heutigen Jenas spricht die Forschung von ca. 14000 Zwangsarbeitern und Kriegsgefangenen sowie zusätzlich $1000 \mathrm{KZ}$-Häftlingen. In Suhl und Altenburg arbeiteten mindestens 8000 , in Gotha mindestens 6500 Menschen unter Zwang.

142 HaAk, Zwangsarbeit (wie Anm. 138), S. 3.

143 Baganz, Volksgemeinschaft (wie Anm. 14), S. 66.

144 Die Geschichte der frühen Konzentrationslager bzw. ,Schutzhaftlager` ist auch Bestandteil einer jüngst erschienenen Studie von Nikolaus Wachsmann, die u. a. durch ihre systematische Aufarbeitung überzeugt. Der Autor zeigt, dass zum Thema eben noch nicht alles gesagt und geschrieben wurde und befindet sich stets auf der Suche nach Erklärungen für das Geschehene; Nikolaus Wachsmann, KL. Die Geschichte der Nationalistischen Konzentrationslager, München 2016. 